\title{
ROLE AND THERAPEUTIC POTENTIAL OF DIETARY KETONE BODIES IN LYMPH VESSEL GROWTH
}

\author{
${ }^{+}$Melissa García-Caballero ${ }^{1,2},{ }^{\dagger}$ Annalisa Zecchin ${ }^{12,8}$, Joris Souffreau ${ }^{1,2}$, Anh-Co Khanh Truong ${ }^{1,2}$, \\ Laure-Anne Teuwen ${ }^{1,2}$, Wesley Vermaelen ${ }^{3,4}$, Rosa Martín-Pérez ${ }^{5,6,8}$, Pauline de Zeeuw ${ }^{1,2}$, Ann Bou- \\ ché $^{1,2}$, Stefan Vinckier ${ }^{1,2}$, Ivo Cornelissen ${ }^{1,2,9}$, Guy Eelen ${ }^{1,2}$, Bart Ghesquière ${ }^{3,4}$, \\ Massimiliano Mazzone ${ }^{5,6}$, Mieke Dewerchin ${ }^{1,2}$ \& Peter Carmeliet ${ }^{1,2,7 *}$
}

\author{
${ }^{1}$ Laboratory of Angiogenesis and Vascular Metabolism, VIB Center for Cancer Biology, VIB, Leuven, B-3000, Belgium. \\ ${ }^{2}$ Laboratory of Angiogenesis and Vascular Metabolism, Department of Oncology, Leuven Cancer Institute, KU Leuven, \\ Leuven, B-3000, Belgium. ${ }^{3}$ Metabolomics Expertise Center, VIB Center for Cancer Biology (CCB), VIB, Leuven, B-3000, \\ Belgium. ${ }^{4}$ Metabolomics Expertise Center, Department of Oncology, KU Leuven, Leuven, B-3000, Belgium. ${ }^{5}$ Laboratory \\ of Tumor Inflammation and Angiogenesis, VIB Center for Cancer Biology (CCB), VIB, Leuven, B-3000, Belgium. ${ }^{6}$ Labora- \\ tory of Tumor Inflammation and Angiogenesis, Department of Oncology, Leuven Cancer Institute, KU Leuven, Leuven, \\ B-3000, Belgium. ${ }^{7}$ State Key Laboratory of Ophthalmology, Zhongshan Ophthalmic Center, Sun Yat-Sen University, \\ Guangzhou, 51006, China. ${ }^{8}$ Present address: Janssen Immunosciences, World Without Disease Accelerator, Pharma- \\ ceutical Companies of Johnson and Johnson, Beerse, B-2340, Belgium. ${ }^{9}$ Present address: Department of Pharmacol- \\ ogy, Research and Development, Janssen Pharmaceutical Company of Johnson and Johnson, Beerse, B-2340, Belgium. \\ $\dagger$ - denotes equal contribution \\ *Correspondence to: \\ Peter Carmeliet, M.D., Ph.D. \\ Laboratory of Angiogenesis and Vascular Metabolism \\ VIB Center for Cancer Biology (CCB) \\ VIB-KU Leuven, Department of Oncology \\ Campus Gasthuisberg O\&N4 \\ Herestraat 49 - B912, \\ B-3000, Leuven, Belgium \\ tel: 32-16-32.30.39 \\ e-mail: peter.carmeliet@kuleuven.vib.be
}




\section{INTRODUCTORY PARAGRAPH}

Lymphatic vessels (LVs), lined by lymphatic endothelial cells (LECs), are indispensable for life ${ }^{1}$. However, the role of metabolism in LECs has been incompletely elucidated. Here, we report that LECspecific loss of OXCT1, a key enzyme of ketone body oxidation ${ }^{2}$, reduces LEC proliferation, migration and vessel sprouting in vitro and impairs lymphangiogenesis in development and disease in Prox $1^{\Delta \mathrm{OXCT1}}$ mice. Mechanistically, OXCT1 silencing lowers acetyl-CoA levels, tricarboxylic acid (TCA) cycle metabolite pools, and nucleotide precursor and dNTP levels, required for LEC proliferation. Ketone body supplementation to LECs induces opposite effects. Notably, elevation of lymph ketone body levels by a high-fat, low-carbohydrate ketogenic diet (HFLC-KD) or by administration of the ketone body $\beta$-hydroxybutyrate $(\beta$-OHB) increases lymphangiogenesis after corneal injury and myocardial infarction. Intriguingly, in a mouse model of microsurgical ablation of LVs in the tail, which recapitulates features of acquired lymphedema in humans, the ketogenic diet improves LV function and growth, reduces infiltration of anti-lymphangiogenic immune cells, and decreases edema, suggesting a novel dietary therapeutic opportunity.

\section{MAIN TEXT}

The lymphatic system is indispensable for life and its dysfunction contributes to various disorders ${ }^{1}$. In particular, insufficient LV formation or function results in lymphedema, a chronic edematous state of the skin involving swelling and fibroadipose tissue deposition in the extremities ${ }^{3}$. Lymphedema is a common post-cancer treatment complication caused by lymphatic injury (surgical removal of lymph nodes) $)^{4,5}$. It is estimated that among cancer patients, 1 in 6 who undergo treatment of breast cancer, melanoma, genito-urinary or gynaecological tumors that involves lymph node removal or radiotherapy will develop secondary lymphedema ${ }^{6,7}$. Despite its medical importance, no approved pharmacological treatment is available, and only symptom-controlling physiotherapy exists. Furthermore, although the importance of LECs in health and disease has been recognized, there are few reports on LEC metabolism ${ }^{8,9}$. 
We recently reported that LECs rely on fatty acid $\beta$-oxidation (FAO) for proliferation, migration and sprouting ${ }^{8}$. Furthermore, FAO facilitates LEC differentiation by generating acetyl-CoA for histone acetylation at lymphangiogenic genes, promoting their expression ${ }^{8}$. Notably, supplementation of acetate (which can be converted to acetyl-CoA) rescues the lymphangiogenesis defect caused by FAO inhibition, highlighting the importance of acetyl-COA as central hub in regulating LV growth $^{8}$. Another source of acetyl-CoA are ketone bodies, energy-rich metabolites secreted by the liver $^{2}$. In extra-hepatic tissues, ketone bodies are oxidized in mitochondria into two molecules of acetyl-CoA (ketone body oxidation; KBO) (Fig. 1a), which can then enter the TCA cycle². In this study, we characterized the role of KBO in lymphangiogenesis.

We first assessed if LECs have access to $\beta$-OHB, the most abundant circulating ketone body in the blood ${ }^{2}$. In adult mice fed a chow diet, $\beta$-OHB was detectable in lymph (collected from the thoracic duct), at similar levels as in plasma (blood collected from the vena cava) (Fig. 1b). To assess whether KBO is active in primary human dermal LECs (HDLECS), we supplemented $\left[\mathrm{U}-{ }^{13} \mathrm{C}\right]-\beta-\mathrm{OHB}$ and measured the incorporation of ${ }^{13} \mathrm{C}$-labeled carbons into acetyl-CoA over time. $\left[\mathrm{U}-{ }^{13} \mathrm{C}\right]-\beta-\mathrm{OHB}$ was rapidly metabolized into acetyl-CoA, yielding a $\mathrm{M}+2$ labeling of $12.5 \%$ after 24 hours (Fig. 1c).

To determine the importance of KBO in HDLECs, we silenced the expression of 3-oxoacidCoA-transferase-1 (OXCT1) (Fig. 1a), a rate-controlling enzyme of $\mathrm{KBO}^{2}$, by transducing HDLECs with lentiviral vectors expressing two different, non-overlapping specific shRNAs against OXCT1, which efficiently lowered its RNA and protein levels (Supplementary Fig. 1a-c). OXCT1 knockdown $\left(\mathrm{OXCT} 1^{\mathrm{KD}}\right.$ ) diminished proliferation and migration of HDLECs (Fig. 1d,e), without affecting HDLEC survival (Supplementary Fig. 1d-f). In addition, OXCT1 $1^{\mathrm{KD}}$ impaired sprouting of HDLEC spheroids, also when HDLECs were mitotically inactivated by mitomycin C (MitoC) to assess cell migration without the impact of proliferation (which contributes to sprouting) (Fig. 1f,g; Supplementary Fig. 1g). Use of the second, non-overlapping shRNA (OXCT1 ${ }^{\mathrm{KD} 2}$ ) yielded similar results (Supplementary Fig. 1h-k). Moreover, silencing of $\beta$-OHB dehydrogenase 1 ( $\mathrm{BDH} 1^{\mathrm{KD}}$ ) (Supplementary Fig. $1 \mathrm{l}$ ), the enzyme that metabolizes $\beta$-OHB to acetoacetate (Fig. 1a), impaired proliferation, migration as well as sprouting 
of HDLECs, phenocopying OXCT1 ${ }^{\mathrm{KD}}$ (Fig. 1h-k; Supplementary Fig. 1m). Conversely, supplementation of ketone bodies ( $\beta$-OHB, acetoacetate) to HDLECs promoted proliferation, migration and sprouting of HDLECs (Supplementary Fig. 1n-r).

To explore whether OXCT1 controls lymphangiogenesis in vivo, we intercrossed Oxct1 $1^{\text {lox/lox }}$ mice $^{10}$ with a LEC-specific tamoxifen-inducible Cre driver line, i.e. Prox1-Cre ${ }^{E R T 2}$ transgenic mice ${ }^{11}$, to generate Cre positive LEC-specific Oxct1-deficient mice (Prox1 $1^{\Delta \mathrm{OxCT1}}$ ) or Cre negative control littermates (Prox ${ }^{\mathrm{WT}}$ ) upon tamoxifen treatment, yielding efficient and broad Oxct1 deletion in LECS (Supplementary Fig. 2a-d). Embryonic lymphangiogenesis was defective in Prox1 ${ }^{\Delta 0 \times C T 1}$ embryos, as evidenced by the presence of edema under the skin and impaired dermal LV growth at E15.5 (Fig. 1l-p). Using the corneal model of injury-induced lymphangiogenesis ${ }^{12}$, we observed that LV area and branch point density were reduced in Prox $1^{\Delta \mathrm{OXCT1}}$ mice (Fig. 2a-c), while CD31+ blood vessel growth was unaffected (Supplementary Fig. 2e). Similar impairment of lymphangiogenesis was observed during the healing phase after myocardial infarction (Fig. 2d-f). Thus, LEC-specific loss of OXCT1 impaired developmental and pathological lymphangiogenesis.

Based on the above findings that KBO promotes lymphangiogenesis, we explored if supplementation of exogenous ketone bodies stimulates LV growth in vivo. We fed wild-type (WT) mice a HFLC-KD, known to increase the levels of circulating ketone bodies ${ }^{2}$, using chow diet as control. Mice fed a HFLC-KD had elevated $\beta$-OHB levels in plasma and lymph (Supplementary Fig. 2f). HFLC-KD caused slight variations in body weight and reduced glucose levels in plasma and lymph (Supplementary Fig. $2 \mathrm{~g}$-i). Since LECs rely also on glucose to sprout ${ }^{9}$, we had expected that the reduction in glucose availability would impair LV growth; however, HFLC-KD stimulated LV growth in the cornea following thermal cauterization, as indicated by the increase in LV area and branch point density (Fig. 2g-i). The number of proliferating BrdU + LECs at the lymphatic front of the corneal LV network was increased upon HFLC-KD (Fig. 2j,k). To confirm that these effects were due to ketone bodies in the HFLC-KD, we supplemented $\beta$-OHB to WT mice via daily intraperitoneal injections and observed a similar increase in lymphangiogenesis (Supplementary Fig. 2j-I). Comparable results were obtained 
in the myocardial infarction mouse model upon feeding a HFLC-KD (Fig. 2I-n). Notably, HFLC-KD or $\beta$-OHB supplementation failed to increase lymphangiogenesis in Prox $1^{\Delta \mathrm{OXCT1}}$ mice, indicating that OXCT1-driven KBO was essential (Fig. 2o,p; Supplementary Fig. 3a-h). Similar results were obtained when supplementing acetoacetate, the substrate of OXCT1 (Supplementary Fig. 3i-j).

In line with the findings that ketone bodies are a source of acetyl-CoA (Fig. 1a), OXCT1 ${ }^{\mathrm{KD}}$ lowered the acetyl-CoA/CoA ratio in HDLECs (Fig. 3a; Supplementary Table 1) and the levels of TCA cycle intermediates (Fig. 3b; Supplementary Table 1). We reported that fatty acid-derived acetylCoA, in conjunction with an anaplerotic substrate, sustains the TCA cycle for deoxynucleotide triphosphate (dNTP) synthesis during proliferation of $E C s^{8,13}$. Similarly, in HDLECs, OXCT1 ${ }^{\mathrm{KD}}$ lowered the cellular pool of dNTPs (Fig. 3c; Supplementary Table 1), paralleling the proliferation defect, and lowered the pool of the nucleotide precursors aspartate and glutamate, as well as the levels of ATP, CTP, GTP and UTP (Fig. 3d; Supplementary Table 1). Similar effects were obtained using OXCT1 ${ }^{\text {KD2 }}$ (Supplementary Fig. 4a-c; Supplementary Table 2). Conversely, ketone body supplementation to HDLECs caused opposite effects, though these effects were modest, likely because these metabolites were already at near-maximal/optimal levels (Fig. 3e-g; Supplementary Table 3). Thus, by sustaining the TCA cycle (presumably in conjunction with an anaplerotic substrate), KBO contributes to dNTP synthesis for DNA replication of HDLECs.

Since the reducing equivalents generated during the conversion of $\beta$-OHB to acetyl-CoA can be delivered to the electron transport chain for ATP production, we measured the oxygen consumption rate $(\mathrm{OCR})$ in HDLECS. OXCT1 $1^{\mathrm{KD}}, \mathrm{OXCT} 1^{\mathrm{KD} 2}$ or $\mathrm{BDH} 1^{\mathrm{KD}}$ modestly (but significantly) reduced oxygen consumption linked to ATP synthesis (OCR ${ }_{\text {ATP }}$ (Fig. 3h, $i$; Supplementary Fig. 4d). Thus, HDLECs can utilize ketone bodies as alternative energy source, even when glucose is available.

To further characterize the use of $\beta$-OHB in HDLECs, we supplemented $\left[\mathrm{U}-{ }^{13} \mathrm{C}\right]-\beta-\mathrm{OHB}$ to HDLECs. Isotopomer analysis revealed ${ }^{13} \mathrm{C}$-label incorporation from $\left[\mathrm{U}-{ }^{13} \mathrm{C}\right]-\beta$-OHB into acetyl-CoA and TCA intermediates (Fig. 3j-k, control bars). When HDLECs were exposed to [U- $\left.{ }^{13} \mathrm{C}\right]-\beta$-OHB for 30 hours to reach steady state, $12.5 \%$ of the ${ }^{13} \mathrm{C}$-label from $\left[\mathrm{U}-{ }^{13} \mathrm{C}\right]-\beta$-OHB was incorporated into acetyl- 
CoA M+2 in control HDLECs, which was reduced upon OXCT1 ${ }^{\mathrm{KD}}$ (Fig. 3j; Supplementary Fig. 4e for $\left.\mathrm{OXCT1}{ }^{\mathrm{KD} 2}\right)$. How a $12 \%$ incorporation of ${ }^{13} \mathrm{C}$-label from $\left[\mathrm{U}-{ }^{13} \mathrm{C}\right]-\beta-\mathrm{OHB}$ into acetyl-CoA suffices to induce the observed metabolic and biological phenotypes, and whether this relates to metabolic pathway compartmentalization, remains to be determined. In accordance, ${ }^{13} \mathrm{C}$-labeling from $\left[\mathrm{U}-{ }^{13} \mathrm{C}\right]-\beta$ OHB into the downstream TCA cycle intermediates citrate, aconitate, $\alpha$-ketoglutarate ( $\alpha \mathrm{KG})$, succinate, fumarate and malate was decreased as well upon OXCT1 ${ }^{\mathrm{KD}}$ in HDLECs (Fig. 3k; Supplementary Fig. $4 \mathrm{f}$ for $\mathrm{OXCT1}{ }^{\mathrm{KD} 2}$ ). Thus, HDLECs metabolize $\beta$-OHB to acetyl-CoA and TCA cycle intermediates.

We also explored if ketone bodies could stimulate lymphangiogenesis not only by sustaining the TCA cycle (in conjunction with other anaplerotic substrates), but also via additional mechanisms. First, ketone bodies are known to have contextual anti-inflammatory effects by directly affecting leukocytes ${ }^{14,15,16}$. In agreement, $\mathrm{CD}^{+}$and $\mathrm{CD} 8^{+} \mathrm{T}$ cell infiltration in lymphedematous mouse tails (see below) was reduced upon HFLC-KD (Fig. 4a,b). Second, we noticed that OXCT1 ${ }^{\mathrm{KD}}$ in HDLECS reduced the expression of lymphangiogenic genes (PROX1, VEGFR-3, VEGF-C, TIE2, ANG1) (Supplementary Fig. 5a-e), while ketone body supplementation elevated expression levels of these genes at the mRNA and protein level (Supplementary Fig. 5f-k).

This raised the question whether ketone bodies are involved in epigenetic regulation of lymphangiogenic gene expression, a plausible mechanism given that LECs utilize FAO-derived acetylCoA to fuel histone acetylation and regulate lymphatic gene expression during LEC differentiation ${ }^{8}$, and that ketone bodies can inhibit histone deacetylases ${ }^{2}$. However, OXCT1 ${ }^{\mathrm{KD}}$ did not alter acetylation of histones H2, H3 and H4 (Supplementary Fig. 5I-left panel), and of H4K8ac (Supplementary Fig. 5I-top right panel). In addition, OXCT1 ${ }^{\mathrm{KD}}$ nor HFLC-KD altered acetylation of histone $\mathrm{H} 3$ at lysine 9 (H3K9ac), a marker of active gene promoters that is sensitive to acetyl-CoA levels ${ }^{17,18}$ (Supplementary Fig. 5l-bottom right panel, m). Thus, ketone bodies did not seem to directly control histone acetylation.

However, we also considered alternative epigenetic mechanisms, since reduced $\alpha$ KG levels and a lower $\alpha \mathrm{KG} /$ succinate ratio upon OXCT1 ${ }^{\mathrm{KD}}$ (Supplementary Fig. 5n,o) could suppress 
transcription by increasing the tri-methylation levels of H3K27 (H3K27me3) ${ }^{19-22}$. Indeed, OXCT1 ${ }^{\mathrm{KD}}$ in HDLECs modestly increased the tri-methylation levels of H3K27 (H3K27me3) (Supplementary Fig. $5 p)$, while tri-methylation of $\mathrm{H} 3 \mathrm{~K} 36$ and $\mathrm{H} 3 \mathrm{~K} 79$ also tended to increase but without reaching statistical significance (not shown). A possible epigenetic effect of ketone bodies requires further study. Third, given the conversion of $\mathrm{NAD}^{+}$to $\mathrm{NADH}$ during ketolysis, we also considered a possible effect of OXCT1 ${ }^{\mathrm{KD}}$ on redox homeostasis, but could not document any change (Supplementary Fig. 5q-t).

We then explored whether the use of HFLC-KD, as a dietary approach, could improve lymphedema. We therefore used a preclinical mouse tail lymphedema model that recapitulates features of secondary lymphedema in humans ${ }^{23,24}$. Notably, in mice fed a HFLC-KD, initiated 2 days after surgical removal of the dermal superficial and deep LVs in the tail, tail swelling was less pronounced (Fig. 4c), with tail volumes being $\pm 50 \%$ lower at 2 and 3 weeks after surgery (Fig. 4d-f). HFLC-KD also reduced dermal thickening (Fig. 4g,h), decreased LV dilation (a hallmark of LV dysfunction $^{25}$ ) and increased the number of LVs (Fig. 4i-k). Also, lymphangiography following intradermal injection of Evans Blue dye revealed higher levels of Evans Blue in the iliac lymph nodes, in the lymph and plasma of mice fed a HFLC-KD as compared to mice fed a chow diet (Fig. 4I-n), implying that HFLC-KD stimulated the formation of new functional LVs across the wound that were able to drain interstitial fluid (and Evans Blue) from the edematous tails. Possibly, the improved drainage and decreased edema could also contribute indirectly to the reduced T cell accumulation mentioned above. Immunoblotting of lymphedematous tail extracts showed increased protein levels of lymphangiogenic VEGFR-3, Tie-2 and Ang1 upon HFLC-KD (Supplementary Fig. 6).

In summary, we demonstrate that ketone bodies stimulate LV growth and function, partly by generating acetyl-CoA to sustain the TCA cycle (likely in conjunction with an anaplerotic substrate) for nucleotide synthesis during LEC replication and by generating reducing equivalents to sustain mitochondrial respiration and ATP production. While not obviously regulating redox homeostasis, ketone bodies may stimulate lymphangiogenesis via additional effects, such as by suppressing the accumulation of anti-lymphangiogenic inflammatory immune cells or by stimulating 
lymphangiogenic gene expression (though a possible epigenetic mechanism requires additional evidence).

Exogenous supplementation of $\beta$-OHB, via ketogenic diet or intraperitoneal injections, stimulates lymphangiogenesis in vivo and ameliorates lymphatic dysfunction and lymphedema. This is noteworthy, given that current treatment of lymphedema is largely symptomatic (including compression garments to reduce swelling, etc.), offering only stabilization and prevention of exacerbation of symptoms, but not providing a cure. A small-size study reported that a ketogenic diet reduced limb volume and improved quality of life in obese patients with limb lymphedema, and ascribed these beneficial effects to weight loss ${ }^{26}$. We report now a molecular explanation for the beneficial effect of ketone bodies. Altogether, our findings may raise interest in using dietary metabolite supplements for treating secondary lymphedema in patients.

AUTHOR INFORMATION: Correspondence and requests for materials should be addressed to PC (peter.carmeliet@kuleuven.vib.be).

ACKNOWLEDGEMENTS: We thank P. Crawford and T. Mäkinen for providing Oxct1 $1^{\text {lox/lox }}$ and Prox1$c r e^{E R T 2}$ mice, respectively. We thank G. Bogaert for providing human foreskins. The authors thank S.M. Fendt for discussion and advice. This work was supported by: fellowships from LE\&RN/FDRS (A.Z.), and supporting grants from IUAP P7/03 (P.C.), Methusalem funding by the Flemish Government (P.C.), FWO (G.0598.12, G.0532.10, G.0817.11, G.0834.13, to P.C.), Leducq Transatlantic Network Artemis (P.C.), AXA Research Fund (1465, to P.C.), Foundation against Cancer (P.C.), Fund for Translation Biomedical Research (FTBO, to P.C.), ERC Advanced Research Grant (EU-ERC269073, to P.C.). We would like to thank, A. Van Nuffelen, A. Carton, A. Manderveld, K. Brepoels, K. Peeters, N. Dai, M. Rifaad, M. Parys, I. Betz, C. De Legher, S. Wyns, P.J. Coolen, M. Nijs, P. Vanwesemael, B. Verherstraeten, G. Dubois, E. Van Dyck, A. Acosta Sanchez and D. Verdegem for their technical assistance, and various laboratory members for their feedback and discussions. 
Author CONTRIBUtions: M.G.C., A.Z., J.S., A-C.K.T., L-A.T., W.V., A.B., R.M.P., S.V., I.C. and B.G. performed research and/or analyzed the data; P.d.Z. managed the collaboration with UZ Leuven, wrote the documentation for the Medical Ethical Committee agreement and the informed consent for HDLEC subjects; M.G.C., A.Z., R.M.P., M.M., G.E., M.D. and P.C. designed experiments; M.G.C., A.Z., and P.C. wrote the paper; P.C. conceptualized and supervised the study. All authors discussed the results and commented on the manuscript.

COMPETING INTERESTS: The authors declare no competing financial or non-financial interests in relation to the work described.

ETHICAL APPROVAL: All applicable international, national, and/or institutional guidelines for the care and use of human samples were followed. All applicable international, national, and/or institutional guidelines for the care and use of animals were followed. All procedures performed in experiments involving animals were in accordance with the ethical standards of the institution and with approval of the institutional ethical committee for animal experimentation. 


\section{LEGENDS TO THE FIGURES}

FIGURE 1: Ketone body oxidation regulates lymphangiogenesis.

a, Scheme of the ketone body oxidation pathway (metabolic enzymes are indicated in green: BDH1, 3-hydroxybutyrate dehydrogenase 1; OXCT1, 3-oxoacid CoA-transferase; mThiolase; citrate synthase); the rate controlling enzyme OXCT1 is boxed. $\mathbf{b}$, LC-MS measurements of $\beta$-OHB levels in mouse plasma and lymph (expressed relative to plasma levels); plasma and lymph were collected from the vena cava and the thoracic duct, respectively ( $n=4$ mice for each condition). $c$, Kinetic analysis of $M+2$ labeling from $\left[\mathrm{U}-{ }^{13} \mathrm{C}\right]-\beta-\mathrm{OHB}$ in acetyl-CoA in HDLECs $(\mathrm{n}=4$ biologically independent samples used in independent experiments). $\mathbf{d},\left[{ }^{3} \mathrm{H}\right]$-thymidine incorporation into DNA in control (ctrl) and OXCT1 $1^{\text {KD }}$ HDLECs ( $n=5$ biologically independent samples used in independent experiments). DPM, disintegrations per minute. e, Scratch wound migration assay using control (ctrl) and OXCT1 $1^{\mathrm{KD}}$ HDLECs ( $n=5$ biologically independent samples used in independent experiments). $\mathbf{f}, \mathbf{g}$, Representative phase contrast images of control and OXCT1 $1^{\mathrm{KD}}$ HDLEC spheroids with and without mitomycin C (MitoC) treatment $(f)$ and quantification of total sprout length $(g)$ ( $n=4$ biologically independent samples used in independent experiments). Scale bars $100 \mu \mathrm{m} . \mathrm{h},\left[{ }^{3} \mathrm{H}\right]$-thymidine incorporation into DNA in control (ctrl) and BDH1 ${ }^{\mathrm{KD}}$ HDLECs ( $\mathrm{n}=5$ biologically independent samples used in independent experiments). DPM, disintegrations per minute. i, Scratch wound migration assay using control and $B D H 1^{K D}$ HDLECs ( $n=5$ biologically independent samples used in independent experiments). $\mathbf{j}, \mathbf{k}$, Representative phase contrast images of control (ctrl) and $\mathrm{BDH} 1^{\mathrm{KD}} \mathrm{HDLEC}$ spheroids with and without mitomycin C (MitoC) treatment (j) and quantification of total sprout length $(k)(n=4$ biologically independent samples used in independent experiments). Scale bars $100 \mu \mathrm{m}$. I, Stereomicrographs of wild-type (Prox1 ${ }^{\mathrm{WT}}$ ) and mutant (Prox1 ${ }^{\triangle \mathrm{OXCT1}}$ ) embryos at E15.5. Asterisks indicate subdermal edema. Representative for $n=14$ Prox $1^{W T}$ embryos and $n=10$ Prox $1^{\Delta 0 x c T 1}$ embryos from 3 independent experiments (3 litters). $\mathbf{m}$, Representative images of the VEGFR3 ${ }^{+}$dermal lymphatic network in E15.5 embryo back skins. White dashed lines delineate the midline gap between LV fronts. Insets to the right are magnifications of the respective boxed areas. Note the abnormal, enlarged LVs in 
Prox $1^{\Delta \mathrm{OXCT1}}$ embryos. Scale bars $500 \mu \mathrm{m}$. n-p, Quantifications of the midline gap size determined by LV outgrowth (n), LV branch point density (expressed as number of branch points per $\mathrm{mm}^{2}$ ) (o) and LV length (expressed in $\mu \mathrm{m})(\mathrm{p})$ per total area analysed ( $\mathrm{n}=14 \operatorname{Prox}^{\mathrm{WT}}$ mice; $\mathrm{n}=10 \operatorname{Prox}^{\Delta 0 \mathrm{O} C T 1}$ mice, from 3 litters). For experiments involving embryos, $50 \mathrm{mg} \mathrm{kg}^{-1}$ tamoxifen was administered to the pregnant dams daily by oral gavage between E8.5 to E10.5 of the pregnancy stage. Data are mean \pm s.e.m. with individual data points shown. ${ }^{*} P<0.05$, ns, not significant, by two-tailed one-sample $t$ test (b) or two-tailed $t$-test (d,e, g-i, k, n-p). Exact $P$-values are as follows: 0.4220 (b); 0.0399 (d); 0.0019 (e); ctrl versus OXCT1 ${ }^{\mathrm{KD}}$ : 0.0094; ctrl versus $\mathrm{OXCT}^{\mathrm{KD}}$ (+ MitoC): <0.0001 (g); 0.0130 (h); 0.0169 (i); ctrl versus BDH1 ${ }^{\mathrm{KD}}$ : 0.0038; ctrl versus $\mathrm{BDH}^{\mathrm{KD}}$ (+ MitoC): <0.0001 (k); 0.0465 (n); 0.0199 (o); 0.0216 (p). 
FIGURE 2: Ketone body oxidation promotes lymphangiogenesis in vivo.

a-c, Representative images of LYVE1 ${ }^{+}$LV (green) and CD31+ blood vessel (red) outgrowth in cauterized corneas of Prox $1^{\mathrm{WT}}$ and Prox $1^{\Delta \mathrm{OXCT1}}$ mice (a), quantification of LV area (b) and LV branch point density (c) (n=13 Prox1 ${ }^{\mathrm{WT}}$ mice; n=14 Prox1 ${ }^{\Delta 0 \mathrm{xcT} 1}$ mice). Scale bars $500 \mu \mathrm{m}$. d-f, Representative images of LYVE1 ${ }^{+}$LVs (green) in infarcted myocardium of Prox $1^{W T}$ and Prox1 $1^{\Delta 0 x c T 1}$ mice (d), quantification of LV area (e) and LV branch point density (f) ( $n=9$ mice for each condition). The white line denotes the circumference of the whole heart. Scale bars $2.5 \mathrm{~mm}$. g-i, Representative images of LYVE1 $^{+}$LV (green) and CD31+ blood vessel (red) outgrowth in cauterized corneas of WT mice receiving a chow diet or HFLC-KD (g), quantification of LV area (h) and LV branch point density (i) ( $n=12$ mice for each condition). Scale bars $500 \mu \mathrm{m}$. j,k, Representative images of $\mathrm{BrdU}^{+}$(red) LYVE1 ${ }^{+}$ (green) LECs in cauterized corneas ( $\mathrm{j}$ ) and density of BrdU $\mathrm{BECs}^{+} \mathrm{k}$ ) in WT mice receiving a chow diet or HFLC-KD ( $n=11$ mice for each condition); confocal images were carefully inspected at different Zplanes to verify that the BrdU ${ }^{+}$cells were LYVE1 $1^{+}$LECs. Scale bars $500 \mu \mathrm{m}$. Insets in (j) are magnifications of the respective boxed areas; arrowheads denote BrdU+ LECs. Scale bars $250 \mu \mathrm{m}$. I-n, Representative images of LYVE1 ${ }^{+} \mathrm{LVs}$ (green) in infarcted myocardium of WT mice receiving a chow diet or HFLC-KD (I), quantification of LV area $(m)$ and LV branch point density $(n)(n=9$ mice for each condition). The white line denotes the circumference of the whole heart. Scale bars $2.5 \mathrm{~mm}$. $\mathbf{0 , p}$, Quantification of corneal (o) or myocardial (p) LV area in Prox $1^{\mathrm{WT}}$ and Prox $1^{\Delta \mathrm{OXCT1}}$ mice fed a chow diet or HFLC-KD upon corneal (o) or myocardial ( $p$ ) injury. In o, $n=13$ mice for Prox $1^{\text {WT }}$ chow; $n=15$ mice for Prox1 $1^{\mathrm{WT}}$ HFLC-KD; $\mathrm{n}=15$ mice for Prox $1^{\triangle \mathrm{OOxCT1}}$ chow and $\mathrm{n}=14$ mice for Prox $1^{\Delta \mathrm{OOXT1}}$ HFLC-KD. In $p, n=9$ mice for all the groups. Mice were fed a chow diet or HFLC-KD for 9 days in the corneal injury model and for 20 days in the myocardial infarction model, initiated the day after the injuries and maintained until the day of sacrifice. Even though the mice used in panels a-f,o,p had a different genetic background (C57BL/6N) and values of the LV area and branch point density were higher than the mice used in panels g-n (C57BL/6J), qualitatively similar data were obtained. LV branch point density in panels $c, f, i, n$ is expressed as number of branch points per $\mathrm{mm}^{2}$. Note: Tamoxifen (50 $\mathrm{mg} \mathrm{kg}^{-1}$ ) was administered for 5 consecutive days to 7-8 week-old Prox $1^{\text {WT }}$ and Prox $1^{\Delta 0 \times c T 1}$ mice 
by oral gavage and experiments were started one week later. Data are mean \pm s.e.m. with individual data points shown. ${ }^{*} P<0.05, \mathrm{~ns}$, not statistically significant, by two-tailed $t$-test $(\mathrm{b}, \mathrm{c}, \mathrm{e}, \mathrm{f}, \mathrm{h}, \mathrm{i}, \mathrm{k}, \mathrm{m}, \mathrm{n})$ or one-way ANOVA multiple comparisons test (o,p). Exact $P$-values are as follows: 0.0341 (b); 0.0448 (c); 0.0035 (e); 0.0056 (f); 0.0114 (h); 0.0375 (i); 0.0308 (k); 0.0375 (m); 0.0480 (n); Prox1 ${ }^{\text {WT }}$ chow versus Prox $1^{\text {WT }}$ HFLC-KD: 0.0389; Prox $1^{\text {WT }}$ chow versus Prox $1^{\triangle \mathrm{OXCT1}}$ chow: 0.0354; Prox $1^{\Delta \mathrm{OXCT1}}$ chow versus Prox1 ${ }^{\triangle 0 x C T 1}$ HFLC-KD: 0.9996 (o); Prox1 ${ }^{\mathrm{WT}}$ chow versus Prox1 ${ }^{\mathrm{WT}}$ HFLC-KD: 0.0002; Prox1 $1^{\mathrm{WT}}$ chow versus Prox $1^{\triangle \mathrm{OXCT1}}$ chow: 0.0282; Prox $1^{\Delta \mathrm{OXCT1}}$ chow versus Prox $1^{\triangle \mathrm{OXCT1}}$ HFLC-KD: 0.7408 ( $p$ ). 
FIGURE 3: Ketone body oxidation contributes to sustain the TCA cycle and dNTP synthesis.

a, Acetyl-COA/CoA ratio in OXCT1 $1^{\mathrm{KD}}$ HDLECs, expressed relative to control HDLECs ( $\mathrm{n}=6$ biologically independent samples used in independent experiments). b, Cellular metabolite pool size normalized for protein content in OXCT1 $1^{\mathrm{KD}}$ HDLECs, expressed relative to control HDLECs ( $\mathrm{n}=8$ biologically independent samples used in independent experiments). Cit, citrate; $\alpha \mathrm{KG}$, alpha-ketoglutarate; Fum, fumarate; Mal, malate. c, Cellular dNTP pool size normalized for protein content in OXCT1 $1^{\mathrm{KD}}$ HDLECs, expressed relative to control HDLECs ( $n=8$ biologically independent samples used in independent experiments). $\mathbf{d}$, Cellular metabolite pool size normalized for protein content in OXCT1 $1^{\mathrm{KD}}$ HDLECs, expressed relative to control HDLECs ( $n=8$ biologically independent samples used in independent experiments). Asp, aspartate; Glu, glutamate. e-g, Acetyl-CoA/CoA ratio (e), and cellular pool size, normalized for protein content, of TCA cycle metabolites, aspartate, glutamate (f), and nucleotides ( $\mathrm{g}$ ) in HDLECs incubated in medium supplemented with $\beta$-OHB (16 mM) or acetoacetate (AcA; $4 \mathrm{mM}$ ), expressed relative to HDLECs in control medium ( $n=6$ biologically independent samples used in independent experiments). Cit, citrate; $\alpha \mathrm{KG}$, alpha-ketoglutarate; Fum, fumarate; Mal, malate. Asp, aspartate; Glu, glutamate. $\mathbf{h}, \mathbf{i}$, Quantification of OCR coupled to ATP synthesis (OCR ATP $_{\text {) }}$ in OXCT1 $1^{\mathrm{KD}} \mathrm{HDLECS}(\mathrm{h})$ and BDH1 $1^{\mathrm{KD}}$ HDLECs (i), relative to control HDLECs ( $\mathrm{n}=5$ biologically independent samples used in independent experiments). $\mathbf{j}$, Analysis of $\mathrm{M}+2$ labeling from $\left[\mathrm{U}-{ }^{13} \mathrm{C}\right]-\beta-\mathrm{OHB}$ into acetyl-CoA in control (ctrl) and OXCT1 ${ }^{\text {KD }}$ HDLECs ( $n=5$ biologically independent samples used in independent experiments). $\mathbf{k}$, Analysis of $\mathrm{M}+2$ labeling from $\left[\mathrm{U}-{ }^{13} \mathrm{C}\right]-\beta-\mathrm{OHB}$ into TCA cycle metabolites in control (ctrl) and OXCT1 $1^{\mathrm{KD}}$ HDLECs ( $\mathrm{n}=5$ biologically independent samples used in independent experiments). $\alpha \mathrm{KG}$, alpha-ketoglutarate. Data are mean \pm s.e.m. with individual data points shown. ${ }^{*} \mathrm{P}<0.05$ by two-tailed one-sample $t$-test $(\mathrm{a}$-i) or two-tailed $t$-test $(\mathrm{j}, \mathrm{k})$. Exact $P$-values are as follows: 0.0125 (a); ctrl versus OXCT1 ${ }^{\mathrm{KD}}$ : 0.0265 (Cit); 0.0069 ( $\alpha \mathrm{KG}$ ); 0.0028 (Fum); 0.0025 (Mal) (b); ctrl versus OXCT1 $1^{\mathrm{KD}}:<0.0001$ (dATP); <0.0001 (dTTP); 0.0017 (dCTP) (c); ctrl versus OXCT1 ${ }^{\mathrm{KD}}$ : 0.0391 (Asp); 0.0491 (Glu); 0.0429 (ATP); 0.0373 (CTP); 0.0093 (GTP); 0.0020 (UTP) (d); ctrl versus supplemented HDLECs: 0.0177 ( $\beta$-OHB); 0.0445 (AcA) (e); ctrl versus supplemented HDLECs: 0.0188, 0.0163 ( $\beta$ OHB, AcA, respectively) (Cit); 0.0214, 0.0424 ( $\beta$-OHB, AcA, respectively) ( $\alpha$ KG); 0.0196, 0.0267 ( $\beta$ - 
OHB, AcA, respectively) (Fum); 0.0398, 0.0082 ( $\beta$-OHB, AcA, respectively) (Mal); 0.0086, 0.0319 ( $\beta$ $\mathrm{OHB}, \mathrm{AcA}$, respectively) (Asp); 0.0324, 0.0479 ( $\beta$-OHB, AcA, respectively) (Glu) (f); ctrl versus supplemented HDLECs: 0.0482, 0.0047 ( $\beta$-OHB, AcA, respectively) (dATP); 0.0387, 0.049 ( $\beta$-OHB, AcA, respectively) (dTTP); 0.076, 0.0310 ( $\beta$-OHB, AcA, respectively) (dCTP); 0.0057, 0.0019 ( $\beta$-OHB, AcA, respectively) (ATP); 0.0011, 0.0027 ( $\beta$-OHB, AcA, respectively) (CTP); 0.0017, 0.0099 ( $\beta$-OHB, AcA, respectively) (GTP); 0.0346, 0.0078 (UTP) (g); 0.0033 (h); 0.0178 (i); 0.0002 (j); <0.0001 (Citrate); 0.0003 (Aconitate); 0.0001 ( $\alpha K G$ ); 0.0229 (Succinate); 0.0003 (Fumarate); <0.0001 (Malate) (k). 
FIGURE 4: Effect of HFLC-KD supplementation on mouse tail lymphedema.

Note: All panels show data obtained in WT mice subjected to surgical excision of tail superficial and deep collecting LVs or sham operation, receiving a chow diet or HFLC-KD.

a,b, Quantification of TCR $\beta^{+}$CD4 ${ }^{+}$cells (a) and TCR $\beta^{+} \operatorname{CD} 8^{+}$cells (b) in the tail ( $n=13$ mice for chow diet and HFLC-KD, $\mathrm{n}=4$ mice for sham). c, Representative images of mouse tails at 21 days (D21). Scale bars $1 \mathrm{~cm}$. d, Percentage of tail volume changes versus day 0 (D0) post-surgery ( $n=6$ independent experiments, with at least 4 mice per group in each experiment). e,f, Quantification of tail volume changes at day 15 (e) and day 21 (f) ( $n=6$ independent experiments, which at least 4 mice per group in each experiment). g, Representative histological H\&E images of tail tissue sections harvested 21 days after LV ablation. The black lines indicate soft tissue thickness. Scale bars $500 \mu m . \mathbf{h}$, Quantification of the soft tissue thickness in mouse tails (D21) ( $n=6$ independent experiments, with at least 4 mice per group in each experiment). i, Representative images of tail tissue sections harvested 21 days after LV ablation and immunostained for LYVE1 (red). Scale bars $500 \mu \mathrm{m}$. Insets at the bottom in (i) are magnifications of the respective boxed area. j, LV lumen size quantification (D21) in mice fed a chow diet or HFLC-KD ( $n=6$ mice and $n=5$ mice for chow and HFLC-KD, respectively). k, LV number quantification per $\mathrm{mm}^{2}$ soft tissue (D21) in mice fed a chow diet or HFLC-KD ( $n=14$ mice for each condition). I-n, Quantification of Evans Blue accumulation in iliac lymph nodes (k), lymph (I) and plasma ( $m$ ) ( $n=16$ mice for chow diet, $n=12$ mice for HFLC-KD, $n=3$ mice for sham groups). Mice were fed a chow or HFLC-KD for 20 days, initiated 2 days after surgical intervention and until their sacrifice. Data are mean \pm s.e.m. with individual data points shown. ${ }^{*} \mathrm{P}<0.05$, ns, not statistically significant, by two-tailed $t$-test. Exact $P$-values are as follows: 0.0134 (a); 0.0098 (b); 0.0351 (day 13); 0.0394 (day 15); 0.0037 (day 21) (d); 0.0394 (e); 0.0037 (f); 0.0098 (h); 0.0366 (j); 0.0178 (k); chow versus HFLC-KD: 0.0385; chow versus HFLC-KD (sham): 0.8995 (I); chow versus HFLC-KD: 0.0030; chow versus HFLC-KD (sham): 0.5351 (m); chow versus HFLC-KD: 0.0019; chow versus HFLC-KD (sham): 0.7021 (n). 


\section{Methods}

CELL CULTURE: Human dermal lymphatic endothelial cells (HDLECS) were isolated from foreskin biopsies (obtained with approval from the Medical Ethical Committee KU Leuven / UZ Leuven (file $\mathrm{nr}$ S57123) and informed consent from all subjects). After dispase digestion to remove the epidermis (12 $\mathrm{h}$ incubation at $4^{\circ} \mathrm{C}$ with $2.5 \mathrm{U} \mathrm{ml}^{-1}$ dispase II) (Sigma-Aldrich), collagenase digestion to harvest all the dermal cells $\left(75 \mathrm{~min}\right.$ incubation at $37^{\circ} \mathrm{C}$ with $0.2 \%$ collagenase I solution; Thermo Fisher Scientific), including endothelial cells, was performed. Immunomagnetic beads (Miltenyi Biotec) were used to isolate $\mathrm{CD} 45^{-} \mathrm{CD} 31^{+}$Podoplanin ${ }^{+}$cells (LECS), which were maintained in Endothelial Cell Growth Medium (EGM)-MV2 (Promocell) ${ }^{27}$. For the in vitro experimental procedures, " $n$ " refers to the number of independent experiments performed on different days, with different, independent batches of HDLECs.

LENTIVIRAL TRANSDUCTIONS: shRNA oligonucleotides to silence OXCT1 (TRCN0000036034: KD; used in the majority of experiments; and TRCN0000036034: KD2; used for confirmatory experiments in Supplementary Figs. 2 and 4) and BDH1 (TRCN0000028460), were cloned into the pLKO-shRNA vector (Sigma-Aldrich) and empty vectors were used as negative controls. Lentiviral particles were produced in 293T cells and were used at a MOI of 20 to transduce HDLECs. Cells were transduced overnight in the presence of $0.5 \mu \mathrm{g} \mathrm{ml}^{-1}$ polybrene and re-fed with fresh medium the next day. Transduced cells were used in functional assays at least 3 to 4 days post-transduction.

RNA ANALYSIS: Total RNA from cell cultures was purified using the PureLink-RNA Mini Kit (Thermo Fisher Scientific) according to the manufacturer's instructions; quality and quantity were measured on a Nanodrop (Thermo Fisher Scientific). cDNA synthesis was performed with the iScript-cDNAsynthesis kit (Bio-Rad). RNA expression analysis was performed by Taqman quantitative RT-PCR using premade primer sets (Thermo Fisher Scientific and IDT; premade primer/probe set identification 
numbers are available upon request). Expression levels were calculated as copies mRNA per 1,000 copies HPRT mRNA.

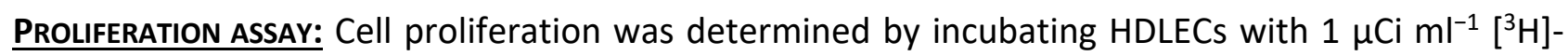
thymidine (Perkin Elmer) for $24 \mathrm{~h}$. Thereafter, cells were fixed with $100 \%$ ethanol for $15 \mathrm{~min}$ at $4^{\circ} \mathrm{C}$, precipitated with $10 \%$ trichloroacetic acid and lysed with $0.1 \mathrm{~N} \mathrm{NaOH}$. The amount of $\left[{ }^{3} \mathrm{H}\right]$-thymidine incorporated into DNA was measured by scintillation counting ${ }^{13}$.

SCRATCH WOUND MIGRATION ASSAY: Scratch wounds were applied on confluent HDLEC monolayers using a $200 \mu \mathrm{l}$ pipette tip and photographed at 0 and $16 \mathrm{~h}$ of incubation in the presence of $500 \mu \mathrm{g} \mathrm{m} \mathrm{l}^{-1}$ mitomycin C (MitoC; Sigma-Aldrich). Migration distance (gap area) was measured with NIH Image J software package and expressed as \% wound closure (gap area at T0 minus gap area at T16 in \% of gap area at T0) ${ }^{13}$.

SPHEROID SPROUTING ASSAY: HDLEC were incubated overnight in hanging drops in EGM-MV2 medium containing methylcellulose (20 volume\% of a $1.2 \%$ solution of methylcellulose 4000 cP) (Sigma-Aldrich) to form spheroids. Spheroids were then embedded in collagen gel and cultured in media supplemented with $100 \mathrm{ng} \mathrm{ml}^{-1}$ recombinant human VEGF-C (Peprotech) for $48 \mathrm{~h}^{8}$. Where indicated, the spheroid sprouting assay was performed with addition of $500 \mu \mathrm{g} \mathrm{ml}^{-1}$ MitoC to the medium upon spheroids embedding in the collagen gel. Spheroids were fixed with $4 \%$ paraformaldehyde (PFA) and phase contrast images were taken with the DMI6000B (Leica) microscope. Quantification of the total sprout length (cumulative length per spheroid) and number of sprouts per spheroid was done using the NIH Image J software package. 
${ }^{13} \mathrm{C}$-TRACER EXPERIMENTS: Cells were incubated in media containing $\left[\mathrm{U}-{ }^{13} \mathrm{C}\right]-\beta-\mathrm{OHB}$ (0.5 mM; Cambridge Isotope Laboratories, Inc.) for $24 \mathrm{~h}$ (steady state), or other indicated time points in the kinetic experiments (Fig. 1c), and samples were processed to analyze tracer incorporation by LC-MS ${ }^{8}$.

METABOLITE POOL AND ISOTOPOMER LABELING ANALYSIS BY LC-MS: Metabolites were extracted from 300,000 cells in $250 \mathrm{ml}$ of a 50:30:20 (methanol:acetonitrile:10 mM Tris, pH 9.3) extraction buffer. Extraction samples were then centrifuged for $5 \mathrm{~min}$ at 20,000 $\mathrm{g}$ and the supernatant was transferred to LC-MS vials. The cell pellet was lysed and used to measure protein levels for normalization purposes. Targeted measurements of acetyl-CoA, coenzyme A, citrate, aconitate, $\alpha$-ketoglutarate, succinate, fumarate, malate, aspartate, glutamate, rNTPs and dNTPs, GSSG, GSH, NAD ${ }^{+}$, NADH, NADP ${ }^{+}$, NADPH were performed using a Dionex UltiMate 3000 LC System (Thermo Fisher Scientific) equipped with a C-18 column (Acquity UPLC-HSST T3 $1.8 \mu \mathrm{m} ; 2.1 \times 150 \mathrm{~mm}$, Waters) coupled to a $\mathrm{Q}$ Exactive Orbitrap mass spectrometer (Thermo Fisher Scientific) operated in negative ion mode. Among the dNTPs, dGTP analysis was not taken into account, since due to co-elution of dGTP with ATP, the dGTP cannot purely be distinguished from ATP by mass. Practically, $5 \mu$ of sample was injected on the Dionex UltiMate 3000 LC System and a step gradient was carried out using solvent A (10 mM tributylamine and $15 \mathrm{mM}$ acetic acid) and solvent B (100\% methanol). The gradient started with $0 \%$ of solvent $B$ and $100 \%$ solvent $A$ and remained at $0 \%$ B until 2 min post injection. A linear gradient to $37 \%$ B was carried out until $7 \mathrm{~min}$ and increased to $41 \%$ until $14 \mathrm{~min}$. Between 14 and 26 min the gradient increased to $100 \%$ of B and remained at $100 \%$ B for 4 min. At 30 min the gradient returned to $0 \% \mathrm{~B}$. The chromatography was stopped at $40 \mathrm{~min}$. The flow was kept constant at $250 \mu \mathrm{min}^{-1}$ at the column was placed at $40^{\circ} \mathrm{C}$ throughout the analysis. The MS operated in full scan - SIM (negative mode) using a spray voltage of $3.2 \mathrm{kV}$, capillary temperature of $320^{\circ} \mathrm{C}$, sheath gas at 10.0, auxiliary gas at 5.0. For Full scan - SIM mode, AGC target was set at 1 e6 using a resolution of 70,000, with a maximum IT of 256 ms. Data collection was performed using Xcalibur software (Thermo Fisher Scientific). 
OXYGEN CONSUMPTION RATE: Cells were seeded at 40,000 cells per well on Seahorse XF24 tissue culture plates (Agilent) the day before the assay. The measurement of oxygen consumption was performed at 6 min intervals ( 2 min mixing, 2 min delay, 2 min measuring) using the Seahorse XF24 analyzer. Inhibitors were used at the following concentrations: oligomycin $(1.2 \mu \mathrm{M})$, antimycin $(1 \mu \mathrm{M})$, FCCP $(5 \mu \mathrm{M})$. The data were normalized to protein content, determined by BCA assay (Thermo Fisher Scientific) upon lysis of the cells (using RIPA buffer) (see below) immediately after the assay ${ }^{28}$.

LDH AsSAY: Cell death was measured by determining the lactate dehydrogenase (LDH) release in the medium using the Cytotoxicity Detection Kit (Roche Applied Science) according to the manufacturer's specifications, whereby low LDH release signifies low cell death and high survival.

APOPTOSIS MEASUREMENTS: cleaved caspase-3 immunostaining: HDLECS present in the medium and trypsinized adherent cells were pooled, then subjected to a centrifuge spin, and resuspended in PBS. From this suspension, aliquots were deposited on slides using a cytospin centrifuge, fixed and stained for cleaved caspase-3 (Cell Signaling) and actin (Alexa fluor 488 phalloidin; Thermo Fisher Scientific); nuclei were stained with Hoechst 33258 (1/1,000 in PBS) ${ }^{28}$. The percentage of apoptotic cells was determined by counting the cleaved caspase $-3^{+}$cell fraction in at least 6 random microscopy fields per HDLEC donor. Annexin V immunocytochemical staining: HDLECs present in the medium and trypsinized adherent cells were pooled, pelleted and resuspended in binding buffer containing $10 \mathrm{mM}$ HEPES, $140 \mathrm{mM} \mathrm{NaCl}, 2.5 \mathrm{M} \mathrm{CaCl}_{2}$ (pH 7.4) at a density of 200,000 cells per $0.1 \mathrm{ml}$. Anti-annexin $\mathrm{V}$ antibody ( $5 \mu \mathrm{l} 0.1 \mathrm{ml}^{-1}$; APC; Thermo Fisher Scientific) was then added at room temperature in the dark for $15 \mathrm{~min}$. After washing, PI (10 $\mathrm{gg} \mathrm{ml}^{-1}$ final concentration; Thermo Fisher Scientific) was added for 5 min prior to the FACS (BD FACSAria III) experiment. Analyses were performed with FlowJo software (FlowJo, LLC). 
KETONE BODY SUPPLEMENTATION IN VITRO: DL- $\beta$-OHB (16 mM; Sigma-Aldrich) and acetoacetate (4 mM; Sigma-Aldrich) were supplemented to a customized media containing $1 \mathrm{mM}$ glucose and $0.6 \mathrm{mM}$ glutamine. The optimal DL- $\beta$-OHB concentration was determined in pilot dose-response experiments; we cannot formally exclude that this high concentration may also induce metabolism-independent signaling. Because acetoacetate is a lithium salt, lithium chloride (Sigma-Aldrich) was included in the control conditions for the acetoacetate treatment.

HISTONE COLLECTION: HDLECs were collected and resuspended in cold nuclear isolation buffer (NIB) (15 mM Tris- $\mathrm{HCl}$ (pH 7.5), $60 \mathrm{mM} \mathrm{KCl,} 15 \mathrm{mM} \mathrm{NaCl}, 5 \mathrm{mM} \mathrm{MgCl}$, $1 \mathrm{mM} \mathrm{CaCl}$, $250 \mathrm{mM}$ sucrose, freshly added: $1 \mathrm{mM} \mathrm{DTT}, 1 \times$ protease inhibitors (Roche), $10 \mathrm{mM}$ sodium butyrate, 0.1\% NP-40). The collected samples were incubated for $30 \mathrm{~min}$ on a rotator at $4^{\circ} \mathrm{C}$ to promote hypotonic swelling of cells and lysis by mechanical shearing during rotation. Nuclei were pelleted at $21,000 \mathrm{~g}$ for $5 \mathrm{~min}$ at $4^{\circ} \mathrm{C}$ and immediately resuspended in $0.4 \mathrm{~N} \mathrm{H}_{2} \mathrm{SO}_{4}$ followed by incubation overnight at $4^{\circ} \mathrm{C}$. After centrifugation at $21,000 \mathrm{~g}$ for $10 \mathrm{~min}$ at $4^{\circ} \mathrm{C}$, histones were precipitated from the supernatant by addition of $20 \%$ trichloroacetic acid for $2 \mathrm{~h}$, followed by centrifugation at $21,000 \mathrm{~g}$ for $10 \mathrm{~min}$ at $4^{\circ} \mathrm{C}$. Pellets were washed twice with acetone. Histone proteins were resuspended in $\mathrm{H}_{2} \mathrm{O}$ and used for immunoblot analysis ${ }^{8}$.

BICINCHONINIC ACID (BCA) ASSAY: Bicinchoninic acid (BCA) assay (Thermo Fisher Scientific) was used to determine protein content. Cells were lysed using RIPA buffer $(20 \mathrm{mM}$ Tris- $\mathrm{HCl}(\mathrm{pH} 7.5)$ containing $150 \mathrm{mM} \mathrm{NaCl}, 1 \mathrm{mM} \mathrm{Na} 2$ EDTA and $1 \mathrm{mM} \mathrm{EGTA}$ ). BCA assay was performed according to the manufacturer's guidelines.

Protein AnAlYSES: Lysates were separated by SDS-PAGE under reducing conditions, transferred to a PVDF membrane, and analyzed by immunoblotting. Primary antibodies used were against OXCT-1 (Proteintech), pan-acetyl lysine (Active Motif), tubulin, GAPDH, histone H3k9ac, histone H4k8ac, 
histone H3k27me3, histone H2, histone H3, histone H4 (Cell Signaling), VEGFR-3, Tie2 (Santa Cruz Biotechnology), and Ang1 (Thermo Fisher Scientific). Membranes were probed with the appropriate HRP-coupled secondary antibody and signal was detected using the ECL system (Amersham Biosciences) according to the manufacturer's instructions. Densitometric analyses were performed using NIH-ImageJ software.

ROS ANALYSIS: Intracellular ROS levels were measured by FACS using 5-(and-6)-chloromethyl-20,70dichlorodihydrofluorescein diacetate, acetyl ester (CM- $\mathrm{H}_{2}$ DCFDA) according to the manufacturer's instructions (Thermo Fisher Scientific). The intracellular ROS levels were determined by pre-incubation of the HDLECs for 30 min with $10 \mathrm{mM} \mathrm{CM}-\mathrm{H}_{2}$ DCFDA and $\mathrm{H}_{2} \mathrm{O}_{2}$ scavenging capacity was determined after a subsequent incubation for $2 \mathrm{~h}$ with $100 \mathrm{mM} \mathrm{H} \mathrm{O}_{2}$ (Merck, Milipore) in serum free M199 medium (Thermo Fisher Scientific). Data were analyzed using FlowJo software (FlowJo, LLC).

MICE: To obtain a tamoxifen-inducible LEC-specific Oxct1 knock-out line, we intercrossed Oxct1 ${ }^{\text {lox/lox }}$ mice $^{10}$ (provided by P. Crawford) with Prox1-Cre ${ }^{\mathrm{ERT} 2}$ mice ${ }^{11}$ (provided by T. Mäkinen) generating Prox1-Cre ${ }^{E R T 2} ; \mathrm{Oxct1}^{\text {lox/lox }} \mathrm{C} 57 \mathrm{BL} / 6 \mathrm{~N}$ mice. Upon subsequent intercrossing with double fluorescent $\mathrm{mT} / \mathrm{mG}$ Cre reporter mice $\mathrm{C} 57 \mathrm{BL} / 6 \mathrm{~J}^{29}$, the resulting Prox ${ }^{\Delta 0 \mathrm{XCT} 1}: \mathrm{mTmG}$ mice allowed determination of recombination efficiency after tamoxifen treatment. Correct Cre-mediated excision of the floxed OXCT1 segment in tamoxifen-treated Prox1 ${ }^{\triangle O X C T 1}$ mice was confirmed via PCR or qRT-PCR analysis of genomic DNA and RNA respectively using standard procedures. Tamoxifen (50 mg kg-1) was administered for 5 consecutive days to 7-8 week-old female mice by oral gavage and experiments were started one week later. For experiments involving embryos, tamoxifen was administered to the pregnant dams daily $\left(50 \mathrm{mg} \mathrm{kg}^{-1}\right.$ ) between E8.5 and E10.5. All experimental procedures were approved by the Institutional Animal Ethics Committee (file nr ECD 116/2014; KU Leuven, Belgium). In all the experimental procedures involving mice, littermate controls were used. 
LEC ISOLATIONS \& RNA EXTRACTION: Murine LECs were isolated from liver, heart, lung and spleen of adult mice ${ }^{30}$. Briefly, mice were anesthetized with ketamine/xylazine (i.p. injection of $100 \mathrm{mg} \mathrm{kg}^{-1}$ body weight of ketamine (Eurovet Animal Health B.V.) and $10 \mathrm{mg} \mathrm{kg}^{-1}$ body weight of xylazine (VMD)) and the perfusion procedure was started once the withdrawal reflex was absent in pelvic limbs. Mice were perfused with $3 \mathrm{ml}$ of PBS followed by organ dissection, and dissociation in $3 \mathrm{ml}$ of a DMEM based digestion buffer containing either $0.2 \%$ collagenase II, $0.2 \%$ collagenase IV (lungs, hearts) or $0.1 \%$ collagenase I, $0.1 \%$ collagenase II (livers), $0.1 \%$ collagenase II, $0.25 \%$ collagenase IV (spleen), dispase II (2.5 $\mathrm{U} \mathrm{ml}^{-1}$ ) (livers and hearts) (Thermo Fisher Scientific), $250 \mathrm{mg} \mathrm{ml}^{-1}$ DNase (Sigma-Aldrich), $2 \mathrm{mM} \mathrm{CaCl}_{2}, 1 \%$ antibiotic/antimycotic, at a perfusion rate of $1 \mathrm{ml} \mathrm{min}^{-1}$. Lungs and hearts were further dissociated using the gentleMACS dissociator system (MACS Technology, Miltenyi Biotec). Next, samples were centrifuged, resuspended in $5 \mathrm{ml}$ of PBS based wash buffer (containing 0.5\% BSA; 2 mM EDTA) and applied to a pre-separation $70 \mu \mathrm{m}$ filter (Corning). The filtered cells were washed twice with wash buffer and ECs were selected using CD31 MicroBeads according to the manufacturer's instructions (MACS Technology, Miltenyi Biotec). Single cell suspensions were stained with a viability dye (eBioscience ${ }^{T M}$ eFluor $^{T M} 450$, Thermo Fisher Scientific), CD45-PeCy7 (eBioscience $^{\text {TM }}$, Thermo Fisher Scientific), podoplanin-APC (BioLegend), CD31-FITC (Thermo Fisher Scientific) at $4^{\circ} \mathrm{C}$ for $30 \mathrm{~min}$. Next, based on antibody staining we FACS sorted (BD FACSAria III) viable, $\mathrm{CD}^{-} 5^{-}, \mathrm{CD} 31^{+}$, podoplanin ${ }^{+} \mathrm{LECS}$, and RNA extracted with the RNeasy Micro Kit (QIAGEN) was converted to cDNA using the SuperScript III First Strand cDNA Synthesis Kit (Thermo Fisher Scientific). RNA expression analysis was performed by Taqman quantitative RT-PCR (Thermo Fisher Scientific).

LYMPH \& PLASMA COLLECTION: Blood and lymph were collected from the vena cava and the thoracic duct, respectively, in anesthetized mice. Samples were then centrifuged at $800 \mathrm{~g}$ for $10 \mathrm{~min}$ to remove the cellular components. 
ANALYSIS OF EMBRYONIC DEVELOPMENT: At E.15.5, the pregnant dams were euthanized by cervical dislocation, and the uteri were removed. Blood flow/viability in embryos was assessed by counting the heart rate through either the yolk sac vessels or directly on the heart using a stereomicroscope. Embryos were dissected and the yolk sacs were washed with PBS and used for genotyping. Stereomicrographs to check the presence/absence of subdermal edema were taken using a LUMAR V12 stereomicroscope (Zeiss).

DEVELOPMENTAL LYMPHANGIOGENESIS: Lymphangiogenesis was assessed by analysis of the anterior dorsal dermal LV network at E15.5 ${ }^{8}$. Briefly, embryos were fixed in 1\% PFA for 10 min before the dorsal skin was dissected and the epidermal and dermal layers were separated under a dissection microscope. Dissected back skins were permeabilized overnight ( $0.5 \%$ Triton X-100, $0.01 \%$ sodium deoxycholate, 1\% BSA, $0.02 \%$ sodium azide), immunostained for VEGFR-3 (R\&D Systems) and images were taken with the LSM780 (Zeiss) confocal microscope. Quantification of the midline gap size, branch point density (number of branch points per $\mathrm{mm}^{2}$ ) and vessel length were performed using NIH ImageJ software.

CORNEAL CAUTERIZATION ASSAY: Corneal lymphangiogenesis was induced by thermal cauterization ${ }^{8}$. After anesthetizing 8-12 week-old female mice with i.p. injection of ketamine $\left(100 \mathrm{mg} \mathrm{kg}^{-1}\right.$ body weight) and xylazine (10 mg kg-1 body weight), the local anesthetic (Unicaïne 0.4\%; Théa Pharma) was applied to the eye and the central cornea was thermally cauterized using an ophthalmic cautery (Optemp II V; Alcon Surgical). Mice were fed chow or high-fat, low-carbohydrate ketogenic diet (HFLC-KD) (94.1\% fat, 1.3\% carbohydrates, 4.6\% protein; Bio-Serv), or injected i.p. with saline or $\beta$ OHB (200 mg kg-1; Sigma-Aldrich) solutions for 9 days (initiated the day after induction of the cornea injury and maintained until sacrifice). For visualization of blood and lymph vessels, cauterized corneas were isolated and whole-mount immunostained for CD31 (BD Biosciences) and LYVE1 (Angiobio), respectively, flat-mounted and imaged using the DMI6000B (Leica) microscope. Analyses of 
the lymphatic area ( $\mathrm{LVVE}^{+}$area in \% of total cornea area) and branch point density (number of branch points per $\mathrm{mm}^{2}$ ) were performed. 5-bromo-2-deoxyuridine (BrdU; Sigma-Aldrich) incorporation was assessed by injecting $\mathrm{BrdU}\left(150 \mathrm{mg} \mathrm{kg}^{-1}\right)$ in the tail vein $2 \mathrm{~h}$ before sacrifice. For detection of histone H3K9ac in lymphatic vessels, cauterized corneas were immunohistochemically stained for VEGFR-3 (R\&D Systems) and H3k9ac (Cell Signaling). Quantitation of the level of H3K9ac fluorescence of LEC nuclei was done using NIH-Image J software. Briefly, the Hoechst $33342^{+} \mathrm{H} \mathrm{KMac}^{+}$nuclei within the lymphatic vessel (VEGFR-3 $3^{+}$area) were individually circled, and the corrected total cell (nucleus) fluorescence $(\mathrm{CTCF}=$ integrated density - (area of selected nucleus $\times$ mean background fluorescence intensity)) was determined per image and expressed in arbitrary units.

MYOCARDIAL INFARCT MODEL: An incision in the third left intercostal space was made in anesthetized mice fixed in a supine position. Thereafter, muscles of the chest wall were prized apart, giving access to the coronary arteries, and a ligation was performed in the center of the descending branch of the left coronary artery. The incision wound was closed and mice were left on a heated surgical table to recover. Mice were fed chow or HFLC-KD or were injected i.p. with saline or with $\beta$-OHB (200 mg $\mathrm{kg}^{-1}$ ) for 20 days (initiated the day after induction of the infarcts and maintained until sacrifice). Whole mount LYVE1 (Angiobio) immunostaining was performed on hearts and they were imaged using the LSM780 (Zeiss) confocal microscope. LYVE' area was quantified using Leica MM AF morphometric analysis software (Leica Microsystems) and expressed as the percentage of the total heart area; branch point density was expressed as the number of branch points per $\mathrm{mm}^{2}$.

LYMPHEDEMA MODEL: Acquired lymphedema was surgically induced in the tails of 8-12 week-old C57BL/6J female mice through a circumferential skin excision made $20 \mathrm{~mm}$ distal to the base of the mouse tail, followed by ablation of the deep and superficial lymphatic vessels ${ }^{23,24}$. For sham controls, only the circumferential skin excision was performed. Mice were fed chow or HFLC-KD, for 20 days (initiated 2 days after surgical removal and maintained until sacrifice). Tail pictures were 
captured at different time points and the edema volume quantified according to the truncated cone formula $\left(V=1 / 3 \cdot \pi \cdot h \cdot\left(R^{2}+R \cdot r+r^{2}\right)\right)^{23,24}$. H\&E staining and LYVE1 (Angiobio) immunostainings were performed on histological tail sections. Soft tissue thickness, and vessel dilation and number of LVs within the soft tissue were quantified using $\mathrm{NIH}$-ImageJ. For the analysis of the immune cell infiltration, edematous tail tissues were collected, epidermis was removed by incubation with dispase (2.5 $\mathrm{U} \mathrm{ml} l^{-1}$ for $60 \mathrm{~min}$ at $\left.37^{\circ} \mathrm{C}\right)$ and dermis was digested $(0.1 \%$ collagenase $\mathrm{II}, 0.25 \%$ collagenase IV using the gentleMACS dissociator system), yielding single cell suspensions that were incubated with a panel of different antibodies for the detection of specific inflammatory markers: viability dye eBioscience $^{\mathrm{TM}}$ eFluor ${ }^{\mathrm{TM}} 450$ (Thermo Fisher Scientific), CD45-FITC (BioLegend), TCR $\beta$-BV421 (BD Biosciences), CD4-PE (BD Biosciences), CD8-APC-Cy7 (BioLegend). Immune infiltrated cells isolated from the lymphedematous tissues were characterized by FACS (BD FACSAria III).

LYMPHANGIOGRAPHY: Lymph, iliac lymph nodes and plasma were collected from mice intradermally injected with Evans Blue ( $200 \mu \mathrm{l}, 0.5 \%$ in PBS; Sigma-Aldrich) at the tip of the tail. The samples were incubated at $55^{\circ} \mathrm{C}$ with formamide (Sigma-Aldrich) for $24 \mathrm{~h}$ followed by absorbance measurements with a microplate spectrophotometer (BioTek).

$\underline{\beta}$-HYDROXYBUTYRATE MEASUREMENTS: Mouse plasma and lymph, collected from the vena cava and the thoracic duct, respectively, were mixed with 50:30:20 (methanol:acetonitrile:10 mM Tris, pH 9.3) extraction buffer (1:10). Extracts were then centrifuged for $5 \mathrm{~min}$ at 20,000 $\mathrm{g}$, the supernatant was transferred to LC-MS vials and $\beta$-OHB levels were analyzed by LC-MS. Isobaric metabolites could not be excluded in our measurements.

STATISTICAL ANALYSIS: Data are represented as mean \pm s.e.m. Statistical significance between two groups was determined by two-tailed $t$-test or two-tailed one-sample $t$-test (Prism version $7.0 \mathrm{~b})$, unless otherwise specified. Statistical significance between multiple groups was determined by 
ANOVA, followed by individual comparisons performed by Bonferroni post-hoc test (Prism version 7.0b). $P<0.05$ was considered statistically significant.

REPORTING SUMMARY: Further information on research design and reagents is available in the Nature Research Reporting Summary linked to this article.

\section{DATA AVAILABILITY}

All data generated or analyzed during this study are included in this published article (and its supplementary information files). The raw data that support the findings of this study are available from the corresponding author upon reasonable request. Supplementary Figures 1, 5 and 6 have associated raw data (uncropped blots) in Supplementary Figure 7. Figure 3 and Supplementary Figure 4 have associated raw data (Excel files) with metabolite abundances.

\section{REFERENCES}

1 Alitalo, K. The lymphatic vasculature in disease. Nat Med 17, 1371-1380, doi:10.1038/nm.2545 (2011).

2 Puchalska, P. \& Crawford, P. A. Multi-dimensional Roles of Ketone Bodies in Fuel Metabolism, Signaling, and Therapeutics. Cell Metab 25, 262-284, doi:10.1016/j.cmet.2016.12.022 (2017).

3 Dayan, J. H., Ly, C. L., Kataru, R. P. \& Mehrara, B. J. Lymphedema: Pathogenesis and Novel Therapies. Annu Rev Med, doi:10.1146/annurev-med-060116-022900 (2017).

4 Avraham, T. et al. Th2 differentiation is necessary for soft tissue fibrosis and lymphatic dysfunction resulting from lymphedema. FASEB J 27, 1114-1126, doi:10.1096/fj.12-222695 (2013).

5 Tabibiazar, R. et al. Inflammatory manifestations of experimental lymphatic insufficiency. PLoS Med 3, e254, doi:10.1371/journal.pmed.0030254 (2006).

6 Cormier, J. N. et al. Lymphedema beyond breast cancer: a systematic review and metaanalysis of cancer-related secondary lymphedema. Cancer 116, 5138-5149, doi:10.1002/cncr.25458 (2010). 
7 Rockson, S. G. \& Rivera, K. K. Estimating the population burden of lymphedema. Ann $N$ Y Acad Sci 1131, 147-154, doi:10.1196/annals.1413.014 (2008).

8 Wong, B. W. et al. The role of fatty acid beta-oxidation in lymphangiogenesis. Nature 542, 49-54, doi:10.1038/nature21028 (2017).

9 Yu, P. et al. FGF-dependent metabolic control of vascular development. Nature 545, 224228, doi:10.1038/nature22322 (2017).

10 Cotter, D. G., d'Avignon, D. A., Wentz, A. E., Weber, M. L. \& Crawford, P. A. Obligate role for ketone body oxidation in neonatal metabolic homeostasis. J Biol Chem 286, 6902-6910, doi:10.1074/jbc.M110.192369 (2011).

11 Bazigou, E. et al. Genes regulating lymphangiogenesis control venous valve formation and maintenance in mice. J Clin Invest 121, 2984-2992, doi:10.1172/JCl58050 (2011).

12 Detry, B. et al. Sunitinib inhibits inflammatory corneal lymphangiogenesis. Invest Ophthalmol Vis Sci 54, 3082-3093, doi:10.1167/iovs.12-10856 (2013).

13 Schoors, S. et al. Fatty acid carbon is essential for dNTP synthesis in endothelial cells. Nature 520, 192-197, doi:10.1038/nature14362 (2015).

14 Pinto, A., Bonucci, A., Maggi, E., Corsi, M. \& Businaro, R. Anti-Oxidant and Anti-Inflammatory Activity of Ketogenic Diet: New Perspectives for Neuroprotection in Alzheimer's Disease. Antioxidants (Basel) 7, doi:10.3390/antiox7050063 (2018).

15 Yamanashi, T. et al. Beta-hydroxybutyrate, an endogenic NLRP3 inflammasome inhibitor, attenuates stress-induced behavioral and inflammatory responses. Sci Rep 7, 7677, doi:10.1038/s41598-017-08055-1 (2017).

16 Goldberg, E. L. et al. beta-Hydroxybutyrate Deactivates Neutrophil NLRP3 Inflammasome to Relieve Gout Flares. Cell Rep 18, 2077-2087, doi:10.1016/j.celrep.2017.02.004 (2017).

17 Henry, R. A., Kuo, Y. M. \& Andrews, A. J. Differences in specificity and selectivity between CBP and p300 acetylation of histone $\mathrm{H} 3$ and H3/H4. Biochemistry 52, 5746-5759, doi:10.1021/bi400684q (2013).

$18 \mathrm{Xu}, \mathrm{Y} . \mathrm{M}$., Du, J. Y. \& Lau, A. T. Posttranslational modifications of human histone H3: an update. Proteomics 14, 2047-2060, doi:10.1002/pmic.201300435 (2014).

19 Peters, A. H. et al. Partitioning and plasticity of repressive histone methylation states in mammalian chromatin. Mol Cell 12, 1577-1589 (2003).

20 Zhang, X., Wen, H. \& Shi, X. Lysine methylation: beyond histones. Acta Biochim Biophys Sin (Shanghai) 44, 14-27, doi:10.1093/abbs/gmr100 (2012).

21 Carey, B. W., Finley, L. W., Cross, J. R., Allis, C. D. \& Thompson, C. B. Intracellular alphaketoglutarate maintains the pluripotency of embryonic stem cells. Nature 518, 413-416, doi:10.1038/nature13981 (2015).

22 TeSlaa, T. et al. alpha-Ketoglutarate Accelerates the Initial Differentiation of Primed Human Pluripotent Stem Cells. Cell Metab 24, 485-493, doi:10.1016/j.cmet.2016.07.002 (2016).

23 Tian, W. et al. Leukotriene B4 antagonism ameliorates experimental lymphedema. Sci Transl Med 9, doi:10.1126/scitranslmed.aal3920 (2017).

24 Zampell, J. C. et al. CD4(+) cells regulate fibrosis and lymphangiogenesis in response to lymphatic fluid stasis. PLoS One 7, e49940, doi:10.1371/journal.pone.0049940 (2012).

25 Gousopoulos, E., Proulx, S. T., Scholl, J., Uecker, M. \& Detmar, M. Prominent Lymphatic Vessel Hyperplasia with Progressive Dysfunction and Distinct Immune Cell Infiltration in Lymphedema. Am J Pathol 186, 2193-2203, doi:10.1016/j.ajpath.2016.04.006 (2016). 
26 Keith, L., Rowsemitt, C. \& Richards, L. G. Lifestyle Modification Group for Lymphedema and Obesity Results in Significant Health Outcomes. American Journal of Lifestyle Medicine 0, 1559827617742108, doi:10.1177/1559827617742108 (2017).

$27 \mathrm{Hu}$, X., Jiang, Z. \& Liu, N. A novel approach for harvesting lymphatic endothelial cells from human foreskin dermis. Lymphat Res Biol 4, 191-198, doi:10.1089/Irb.2006.4403 (2006).

28 Vandekeere, S. et al. Serine Synthesis via PHGDH Is Essential for Heme Production in Endothelial Cells. Cell Metab, doi:10.1016/j.cmet.2018.06.009 (2018).

29 Muzumdar, M. D., Tasic, B., Miyamichi, K., Li, L. \& Luo, L. A global double-fluorescent Cre reporter mouse. Genesis 45, 593-605, doi:10.1002/dvg.20335 (2007).

30 Kalucka, J. et al. Quiescent Endothelial Cells Upregulate Fatty Acid beta-Oxidation for Vasculoprotection via Redox Homeostasis. Cell Metab, doi:10.1016/j.cmet.2018.07.016 (2018). 